\title{
Research Article \\ Diameter Control and Photoluminescence of ZnO Nanorods from Trialkylamines
}

\author{
Tamar Andelman, Yinyan Gong, Gertrude Neumark, and Stephen O'Brien \\ Department of Applied Physics and Applied Mathematics, Materials Research Science and Engineering Center, \\ Columbia University, 500 W 120th Street, New York, NY 10027, USA
}

Received 16 February 2007; Accepted 25 April 2007

Recommended by Ping Xiao

A novel solution method to control the diameter of $\mathrm{ZnO}$ nanorods is reported. Small diameter (2-3 nm) nanorods were synthesized from trihexylamine, and large diameter $(50-80 \mathrm{~nm})$ nanorods were synthesized by increasing the alkyl chain length to tridodecylamine. The defect (green) emission of the photoluminescence (PL) spectra of the nanorods varies with diameter, and can thus be controlled by the diameter control. The small $\mathrm{ZnO}$ nanorods have strong green emission, while the large diameter nanorods exhibit a remarkably suppressed green band. We show that this observation supports surface oxygen vacancies as the defect that gives rise to the green emission.

Copyright (c) 2007 Tamar Andelman et al. This is an open access article distributed under the Creative Commons Attribution License, which permits unrestricted use, distribution, and reproduction in any medium, provided the original work is properly cited.

\section{INTRODUCTION}

Zinc oxide is a widely studied material, both for its many applications, which range from optoelectronic devices to rubber additives, and for its numerous possible nanocrystal morphologies, including spherical nanoparticles [1, 2], nanotetrapods [3], nanotriangles [4], and nanorods [5-8]. The nanorod morphology in particular shows promise for use in nanolasers [9], photovoltaic devices [10], and field effect transistors [11]. Control over rod length and diameter is important for such applications. There are currently several solution-based methods for controlling nanorod diameter [12-15]. We report here a simple solution method to control the diameter of $\mathrm{ZnO}$ nanorods by increasing the chain length of the amine solvent used. The PL data shows that the green emission varies with nanorod diameter, thus diameter control can be an effective method to control green emission [16]. This is of interest for the application of $\mathrm{ZnO}$ nanorods for UV lasers, where the UV emission is important and the green emission is unwanted.

\section{EXPERIMENT}

$\mathrm{ZnO}$ nanorods of controllable diameter were prepared by a modified version of our previously reported synthesis [5]. The precursor, zinc acetate dihydrate, the capping agent oleic acid (OA), and one of the solvents trihexylamine (THA) or tridodecylamine (TDDA) were mixed and heated to synthesize nanorods of different diameters. The nanocrystals were characterized by X-ray diffraction (XRD) and transmission electron microscopy (TEM). Photoluminescence (PL) spectroscopy was performed to study the optical properties of the nanorods.

\section{MATERIALS AND METHODS}

Synthesis: precursor zinc acetate dihydrate $\left(\mathrm{ZnAc}_{2} \cdot 2 \mathrm{H}_{2} \mathrm{O}\right)$, capping ligand oleic acid, technical grade $90 \%$ were mixed in a $1: 1$ ratio in a three-neck flask in the solvent of choice: trihexylamine $96 \%$, tridecylamine $98 \%$, or tridodecylamine $85 \%$. All chemicals were purchased from Aldrich and used without further purification. In a typical synthesis, $1.4 \mathrm{~g}$ of the precursor was combined with $2 \mathrm{~mL}$ oleic acid in $15 \mathrm{~mL}$ of an amine solvent and heated at $180^{\circ} \mathrm{C}$ under vacuum for 30 minutes to remove any water from the system. The solution was then heated under nitrogen to $286-300^{\circ} \mathrm{C}$, and turned cloudy white when the nanorods formed. After 5-10 minutes, the heating was stopped. Upon cooling, the solution was centrifuged, and the nanorods washed repeatedly and redispersed in hexane. XRD: an inel multipurpose diffractometer was used. Samples were prepared by drop casting the nanocrystal solution onto a silicon wafer. 
TEM: JEOL 100 CX was used. Samples were prepared by placing a drop of the nanocrystal solution on a 400 mesh carbon grid with Formvar.

PL: PL was recorded at room temperature, using the $325 \mathrm{~nm}$ emission from a He-Cd laser (the maximum excitation intensity, $\mathrm{I}_{\max } \approx 10 \mathrm{~W} / \mathrm{cm}^{2}$ ). The optical absorption spectra were recorded with an Agilent HP8453 spectrometer. For optical characterization, all samples were isolated from their growth solution, redispersed in hexane and kept in a quartz cell.

\section{RESULTS AND DISCUSSION}

The XRD pattern of each nanorod sample matches the standard bulk wurtzite structure, (P63 mc, JCPDF no. 36-1451), as shown in Figure 1. The intensity of the peaks in the nanorods from trihexylamine, 2-3 nm diameter, (sample (a)) differs greatly from the bulk due to the larger number of planes along the long axis of the rod as compared to the small diameter. This indicates that the growth direction of the rod is along the $c$ axis. This is similar to our previous result of $\mathrm{ZnO}$ nanorods synthesized from trioctylamine [5]. The diameter of the nanorods from tridodecylamine is larger, 50-80 nm (sample (b)), and the relative intensity of the nanorods begins to resemble that of the standard bulk pattern.

Figure 2 shows the TEM images of the nanorods prepared from the different tertiary amine solvents (on the left), along with the corresponding PL spectra (on the right). The $\mathrm{ZnO}$ nanorods synthesized from trihexylamine, Figure 2 (a), measure $2-3 \mathrm{~nm}$ in diameter and 60-75 $\mathrm{nm}$ in length. Lengths can be varied by increasing or decreasing reaction time.

These nanorods are quite similar to those synthesized previously from trioctylamine [5]. The nanorods from tridodecylamine, Figure 2 (b) were much larger, measuring 50$80 \mathrm{~nm}$ in diameter and 200-400 $\mathrm{nm}$ in length. The product of the synthesis from tridecylamine, an intermediate chain length solvent, was not discreet nanorods. Rather, the resulting nanomaterial was crystalline by XRD but of poorly defined morphology.

Our previous experiments with morphological control of $\mathrm{ZnO}$ nanoparticles led us to understand that the solvent plays a crucial role in the nanoparticle growth [4]. It seems that the tertiary amine is a strong coordinating solvent, and acts as a second ligand to stabilize and quench growth in the radial direction [17]. The forming nanocrystal then grows quickly from the supersaturated solution along its fast growth direction, the polar [0001] axis, which results in the elongated shape of a rod. When the solvent tertiary amine carbon chain length is increased, the diameter of the resulting nanorod is larger. Although the longer chain tertiary amines are still coordinating, they are bulkier and more sterically hindered, and thus do a poorer job of quenching the radial growth. This results in larger diameter nanorods. Thus, the nanorods synthesized from trihexylamine and trioctylamine are $2-3 \mathrm{~nm}$ in diameter, while those synthesized from tridodecylamine are $50-80 \mathrm{~nm}$ in diameter. It has been observed in other nanocrystal systems that increasing the chain length

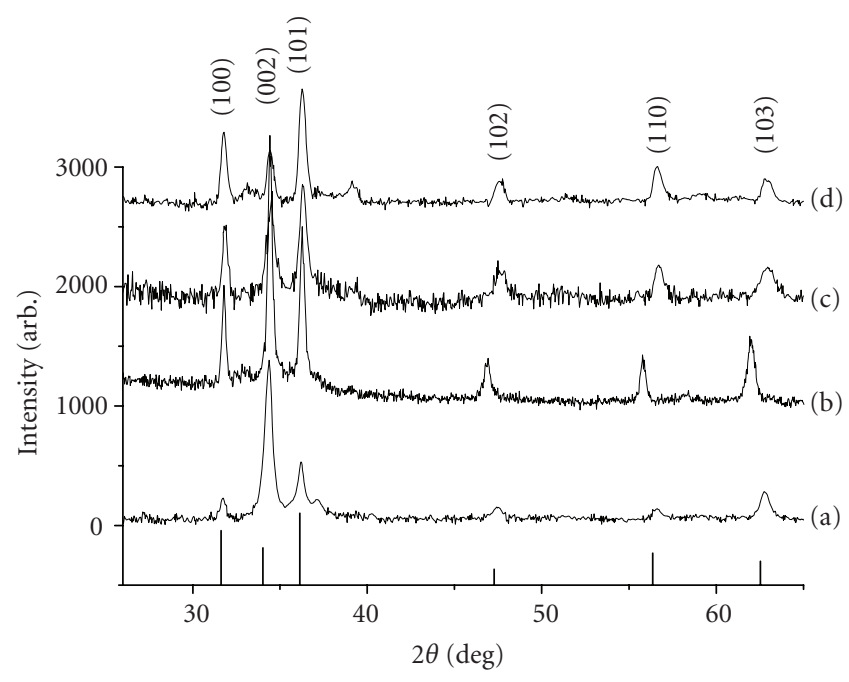

FIGURE 1: XRD spectra of zinc oxide nanorods synthesized from (a) trihexylamine, 2-3 nm diameter, (b) tridodecylamine, 50-80 nm diameter, with bulk relative intensity indicated by straight lines.

of the ligand increased the nanoparticle size [18]: Prasad et al. postulate that there exists an optimum particle size which allows for the most stable packing of the capping agent, with longer ligands prefer less curved surfaces, resulting in larger nanoparticles. In Figure 2, on the right, we show the room temperature photoluminescence (black circles) and optical absorption (inset) spectra of $\mathrm{ZnO}$ nanorods with 2 and 50$80 \mathrm{~nm}$ in diameter. The absorption spectra (Figure 2 (a) inset and, Figure 2 (b) inset) of both samples exhibit strong excitonic absorption, with the peaks showing a red shift with increasing nanorod diameter. The peak positions (indicated by the arrows) are at $3.50 \pm 0.05 \mathrm{eV}$, and $3.31 \pm 0.03 \mathrm{eV}$, respectively. The first value is much larger than the excitonic absorption edge of bulk $\mathrm{ZnO}$, which indicates a strong quantum confinement effect. The value observed here is in agreement with that previously reported $[5,18]$. The PL of both nanorod samples shows a near band edge UV emission accompanied by a broad green emission. The relative intensity of the green emission is reduced with the increase of the nanorod diameters. It is generally accepted that the observed visible luminescence is due to surface states, $[4,16,19,20]$. In our previous studies [4], we found that for $\mathrm{ZnO}$ nanocrystals with different morphologies, the relative intensity of the green emission is quenched with the decrease of the surfaceto-volume ratio. The association of surface states to the green emission is further supported by the polarized luminescence. It has been observed [20] that the intensity of the green emission has its maximum intensity along the direction perpendicular to the $c$-axis, while the UV emission has its maximum intensity along the $c$-axis. Here, the observed reduction of green emission intensity with decreased surface/volume ratio is consistent with the attribution of green luminescence to the surface states. Control over the nanorod diameter therefore provides control over the intensity of the green emission. 

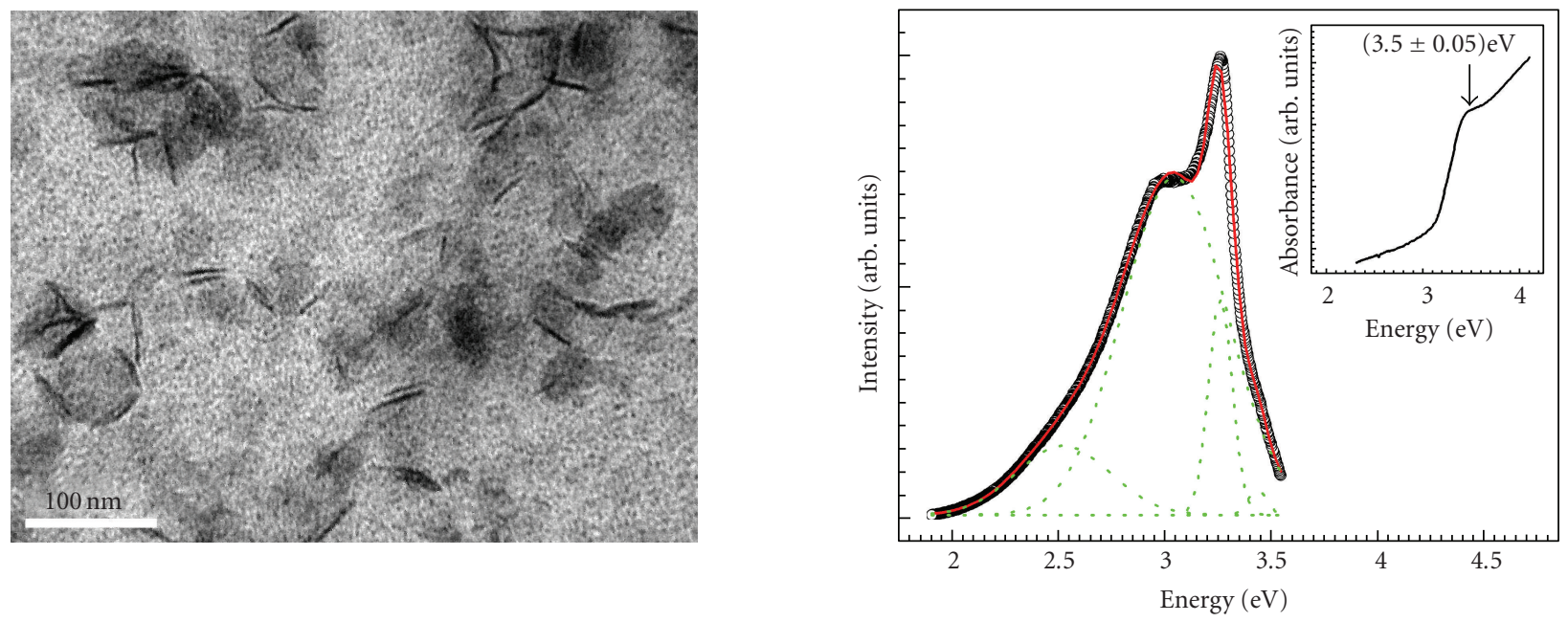

(a)
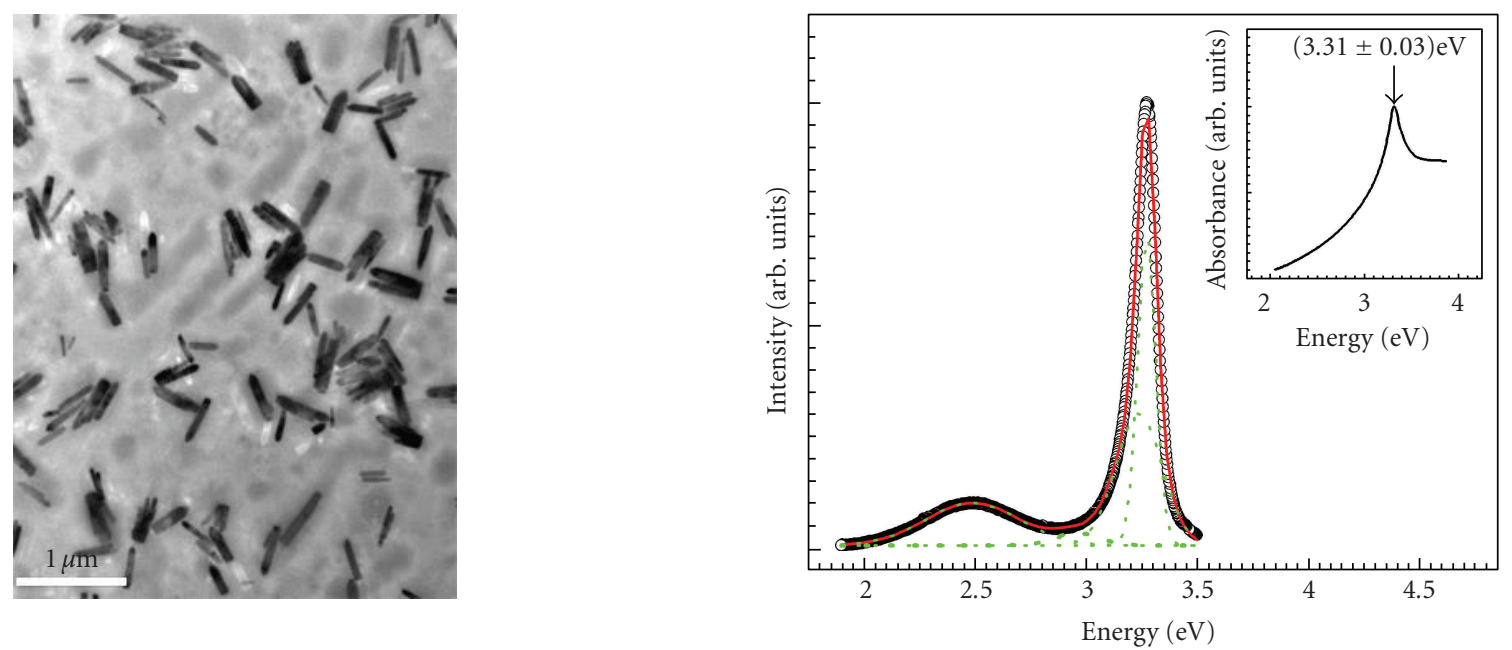

(b)

FIGURE 2: TEM images (left side) and corresponding photoluminescence (black circles) and optical absorption (inset) spectra (right side) of $\mathrm{ZnO}$ spectra of zinc oxide nanorods synthesized from (a) trihexylamine, $2 \mathrm{~nm}$ diameter (b) tridodecylamine, 50-80 nm diameter. The PL spectra were fitted by Gaussian functions (shown as red solid line and green dotted lines).

\section{ACKNOWLEDGMENTS}

This work was supported primarily by the MRSEC program of the National Science Foundation under award number DMR-0213574, and NSF-CAREER award, DMR-0348938, and by the New York State Office of Science, Technology, and Academic Research (NYSTAR). Partial support was appreciated from the U.S. Department of Energy, Office of Basic Energy Sciences, through the Catalysis Futures grant DE-FG0203ER15463. The first author is grateful for support from the NSF Graduate Research Fellowship.

\section{REFERENCES}

[1] E. Wong, J. E. Bonevich, and P. C. Searson, "Growth kinetics of nanocrystalline $\mathrm{ZnO}$ particles from colloidal suspensions," Journal Physical Chemistry B, vol. 102, no. 40, pp. 7770-7775, 1998.

[2] E. A. Meulenkamp, "Synthesis and growth of $\mathrm{ZnO}$ nanoparticles," Journal of Physical Chemistry B, vol. 102, no. 29, pp. 5566-5572, 1998.

[3] H. Yan, R. He, J. Pham, and P. Yang, "Morphogenesis of onedimensional $\mathrm{ZnO}$ nano- and microcrystals," Advanced Materials, vol. 15, no. 5, pp. 402-405, 2003. 
[4] T. Andelman, Y. Gong, M. Polking, et al., "Morphological control and photoluminescence of zinc oxide nanocrystals," Journal of Physical Chemistry B, vol. 109, no. 30, pp. 14314-14318, 2005.

[5] M. Yin, Y. Gu, I. L. Kuskovsky, et al., "Zinc oxide quantum rods," Journal of the American Chemical Society, vol. 126, no. 20, pp. 6206-6207, 2004.

[6] B. Liu and H. C. Zeng, "Hydrothermal synthesis of $\mathrm{ZnO}$ nanorods in the diameter regime of $50 \mathrm{~nm}$," Journal of the American Chemical Society, vol. 125, no. 15, pp. 4430-4431, 2003.

[7] M. Lai and D. J. Riley, "Templated electrosynthesis of zinc oxide nanorods," Chemistry of Materials, vol. 18, no. 9, pp. 22332237, 2006.

[8] S. C. Lyu, Y. Zhang, C. J. Lee, H. Ruh, and H. J. Lee, "Lowtemperature growth of $\mathrm{ZnO}$ nanowire array by a simple physical vapor-deposition method," Chemistry of Materials, vol. 15, no. 17, pp. 3294-3299, 2003.

[9] M. H. Huang, S. Mao, H. Feick, et al., "Room-temperature ultraviolet nanowire nanolasers," Science, vol. 292, no. 5523, pp. 1897-1899, 2001.

[10] P. Ravirajan, A. M. Peiró, M. K. Nazeeruddin, et al., "Hybrid polymer/zinc oxide photovoltaic devices with vertically oriented $\mathrm{ZnO}$ nanorods and an amphiphilic molecular interface layer," Journal Physical Chemistry B, vol. 110, no. 15, pp. 76357639, 2006.

[11] B. Sun and H. Sirringhaus, "Solution-processed zinc oxide field-effect transistors based on self-assembly of colloidal nanorods," Nano Letters, vol. 5, no. 12, pp. 2408-2413, 2005.

[12] Y. Tak and K. Yong, "Controlled growth of well-aligned $\mathrm{ZnO}$ nanorod array using a novel solution method," Journal Physical Chemistry B, vol. 109, no. 41, pp. 19263-19269, 2005.

[13] Q. Li, V. Kumar, Y. Li, H. Zhang, T. J. Marks, and R. P. H. Chang, "Fabrication of $\mathrm{ZnO}$ nanorods and nanotubes in aqueous solutions," Chemistry of Materials, vol. 17, no. 5, pp. 10011006, 2005.

[14] B. Liu and H. C. Zeng, "Room temperature solution synthesis of monodispersed single-crystalline $\mathrm{ZnO}$ nanorods and derived hierarchical nanostructures," Langmuir, vol. 20, no. 10, pp. 4196-4204, 2004.

[15] A. Dev, S. K. Panda, S. Kar, S. Chakrabarti, and S. Chaudhuri, "Surfactant-assisted route to synthesize well-aligned $\mathrm{ZnO}$ nanorod arrays on sol-gel-derived $\mathrm{ZnO}$ thin films," Journal Physical Chemistry B, vol. 110, no. 29, pp. 14266-14272, 2006.

[16] I. Shalish, H. Temkin, and V. Narayanamurti, "Size-dependent surface luminescence in $\mathrm{ZnO}$ nanowires," Physical Review B, vol. 69, Article ID 245401, 4 pages, 2004.

[17] X. M. Sun, X. Chen, Z. X. Deng, and Y. D. Li, "A CTAB-assisted hydrothermal orientation growth of $\mathrm{ZnO}$ nanorods," Materials Chemistry and Physics, vol. 78, no. 1, pp. 99-104, 2003.

[18] B. L. V. Prasad, S. I. Stoeva, C. M. Sorensen, and K. J. Klabunde, "Digestive ripening of thiolated gold nanoparticles: the effect of alkyl chain length," Langmuir, vol. 18, no. 20, pp. 7515-7520, 2002.

[19] Y. Gu, I. L. Kuskovsky, M. Yin, S. O’Brien, and G. Neumark, "Quantum confinement in $\mathrm{ZnO}$ nanorods," Applied Physical Letter, vol. 85, no. 17, pp. 3833-3835, 2004.

[20] C. L. Yang, J. N. Wang, W. K. Ge, L. Guo, S. H. Yang, and D. Z. Shen, "Enhanced ultraviolet emission and optical properties in polyvinyl pyrrolidone surface modified $\mathrm{ZnO}$ quantum dots," Journal of Applied Physics, vol. 90, no. 9, pp. 4489-4493, 2001. 

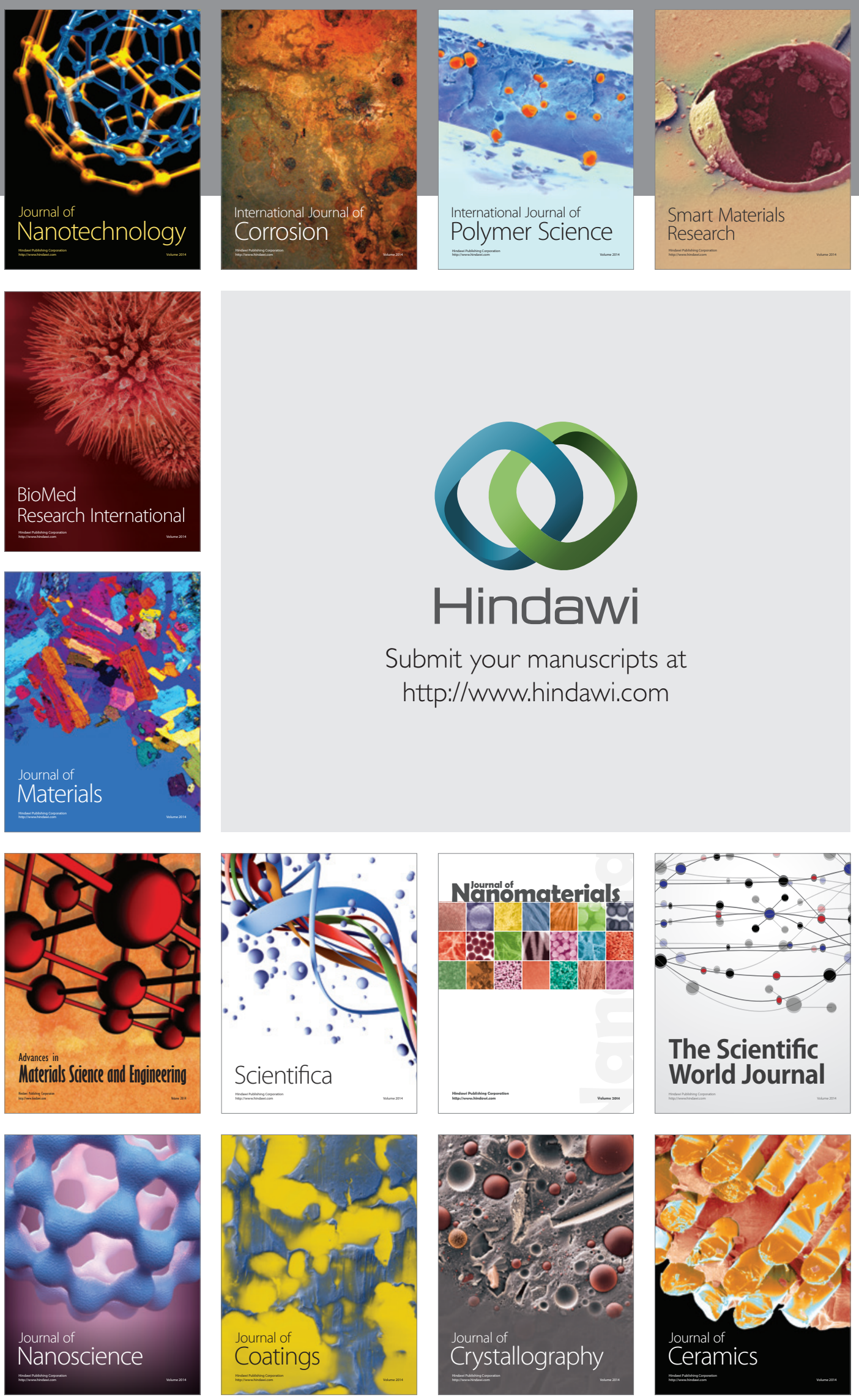

The Scientific World Journal

Submit your manuscripts at

http://www.hindawi.com

\section{World Journal}

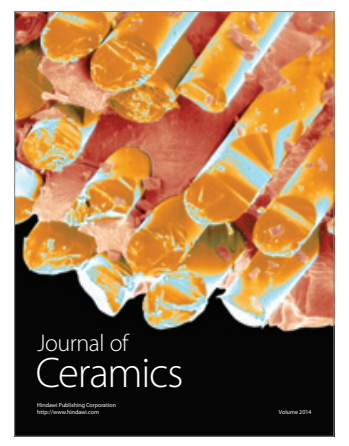

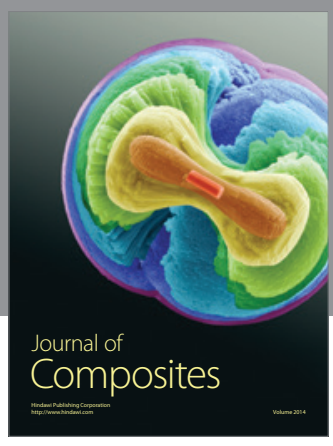
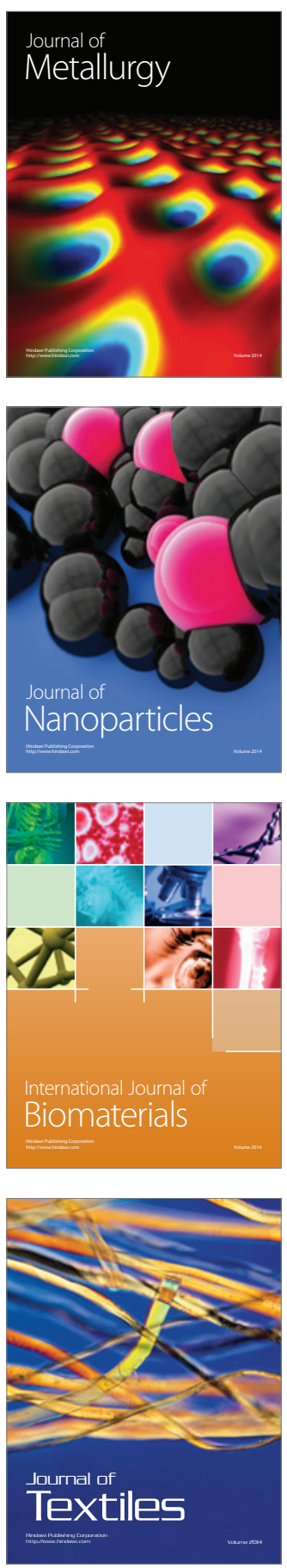